\title{
Microbiological effects of an antiseptic mouthrinse in irradiated cancer patients
}

\author{
Isabel Lanzós ${ }^{1}$, David Herrera ${ }^{2}$, Sagrario Santos ${ }^{1}$, Ana O'Connor ${ }^{3}$, Carmen Peña ${ }^{4}$, Eduardo Lanzós ${ }^{5}$, Ma- \\ riano $\operatorname{Sanz}^{2}$
}

\footnotetext{
${ }^{1}$ Master in Periodontology, Faculty of Odontology, University Complutense, Madrid, Spain

${ }^{2}$ Full-time Professor, ETEP Research Group, University Complutense, Madrid, Spain

${ }^{3}$ Laboratory Technician. Faculty of Odontology, University Complutense, Madrid, Spain

${ }^{4}$ Adjunct Physician, Oncology Radiotherapy Service, Hospital 12 de Octubre, Madrid, Spain

${ }^{5}$ Head of Service, Oncological Radiotherapy Service, Hospital 12 de Octubre, Madrid, Spain
}

Correspondence:

Faculty of Odontology

Universidad Complutense

Plaza Ramón y Cajal, s/n

28040 Madrid SPAIN

davidher@odon.ucm.es

Received: 02/08/2010

Accepted: 14/11/2010

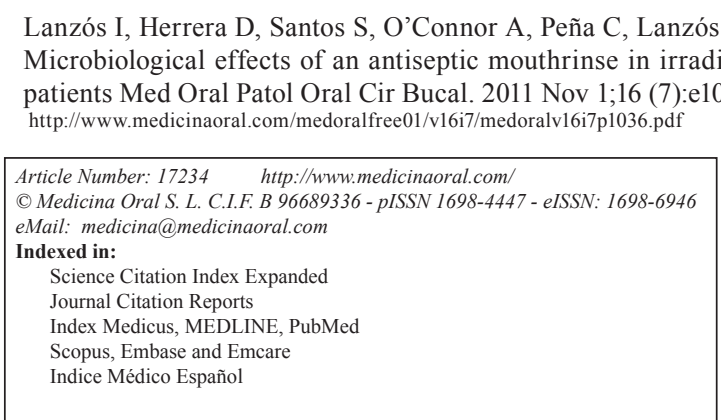

\begin{abstract}
Objective: To assess the microbiological effects of an antiseptic, non-alcohol based mouth-rinse containing chlorhexidine and cetylpyridinium chloride, in patients undergoing radiation therapy for head-and-neck cancer.

Study Design: This was a parallel, double-blind, prospective, randomized clinical trial, including patients irradiated as part of the therapy of head-and-neck cancer, aged 18-75, with at least 10 teeth, and willing to sign an informed consent. Cancer patients were randomly assigned to one of the two treatments (test mouth-rinse or a placebo). Three visits were scheduled (baseline, 14 and 28 days). Microbiological findings were evaluated in tongue, mucosa and subgingival samples, by means of culture. Microbiological variables were assessed by means of the Mann-Whitney, Wilcoxon and chi-square tests.

Results: 70 patients were screened and 36 were included. The detection of Candida species in mucosa and tongue samples showed significant reductions in the test group. Total bacterial counts decreased in both groups from baseline to the 2-week visit, while minor changes occurred between 2 and 4 weeks (effects on $P$. gingivalis, $P$. intermedia, C. rectus, E. corrodens).

Conclusions: Within the limitations of the small sample size, this study suggests that the use of the tested mouthrinse may lead to improvements in microbiological parameters in patients irradiated for head-and-neck cancer.
\end{abstract}

Key words: Mucositis, head-and-neck tumour, radiotherapy, chlorhexidine, cetyl-pyridinium chloride, microbiology. 


\section{Introduction}

Oral mucositis and xerostomia are the most common complications in patients undergoing non-surgical therapy of cancer (1). Changes in quantity and/or quality of saliva may make difficult for the patient to eat or to use dentures, and it also leads to changes in the oral microbiology. The reductions in $\mathrm{pH}$ and in the buffer capacity may favour the overgrowth of opportunistic species $(2,3)$.

There is a scarcity of data on the effects of radiotherapy in the oral microbiota. In irradiated patients a higher prevalence of Candida species colonization in the oral cavity was reported, when compared to healthy controls (4). A study from Leung et al. (3) found that the subgingival microflora from shallow pockets in irradiated patients was similar to that of gingivitis in systemically healthy patients, but with a tendency to show the overgrowth of non-typical oral species, including fungi. When irradiated patients were evaluated 6 months after cancer therapy (5), a transient colonization with aerobic and anaerobic facultative rods and gram-negative cocci was observed. Six to eight months after therapy, Candida sp. and especially enterics were more frequently detected in previously irradiated patients, as compared with controls (6). Other studies have focussed on the changes in caries-associated microflora. One group reported a high incidence of caries "radiation caries", due to the long-term radiotherapy-induced changes in the microflora (7). In another study, an overgrowth of Streptococcus mutans was not observed, but other nonmutans streptococci were isolated (8), in contrast to another study in children aged 4-15 years, which reported significantly higher $S$. mutans counts as compared with controls (9).

Numerous studies in systemically healthy patients have demonstrated that chlorhexidine (CHX) can reduce bacterial and mycotic colonization of the oral cavity (10).

Different CHX formulations have been recently introduced in the market with the aim of reducing its side effects (for example, by eliminating alcohol from the formulations) or to increase its antimicrobial activity (for example, by adding new active agents). However, these new formulations must demonstrate the bio-availability of CHX and therefore, its antimicrobial activity. One of these new formulations lacking alcohol content and combined with an additional active agent (cetyl-pyridinium chloride, CPC), has been marketed (PerioAid Tratamiento ${ }^{\circledR}$ and PerioAid Mantenimiento ${ }^{\circledR}$, Dentaid, Cerdanyola del Vallés, Spain) and proved to be both safe and effective, at standard $0.12 \%$ concentration $(11,12)$ or even at a lower concentration $(0.05 \%)(13,14)$.

In summary, radiotherapy for HNT induces important oral side effects, such as xerostomia and mucositis, and these changes may favour mucosal and saliva colonization by opportunistic microorganisms (Candida sp.), as well as the overgrowth of anaerobic bacterial species, with the subgingival flora as primary niche. Our hypothesis is that the use of an effective antiseptic mouth rinse (combining $\mathrm{CHX}$ and $\mathrm{CPC}$ ) would prevent this overgrowth and may help to maintain a more healthrelated flora in the mouth. It is, therefore, the objective of investigation was to assess the microbiological effects of an antiseptic, non-alcohol based, mouth rinse containing $\mathrm{CHX}$ and $\mathrm{CPC}$, in head-and-neck cancer patients under irradiation therapy. Clinical results have described in a previous paper (15)

\section{Patients and Method \\ Patients}

A total of 70 Consecutive patients were selected at the Oncology Radiotherapy Service at the "12 de Octubre" Hospital (Madrid), using the criteria already described in Lanzos et al. (15), including patients irradiated as part of the therapy of head-and-neck cancer, aged 18-75, with at least 10 teeth, and willing to sign an informed consent. Patients were excluded if they were already diagnosed of suffering a mucosal pathology, such as lichen or lupus.

Finally, 36 patients (32 male and 4 female patients) were included. All suffered from head-and-neck cancer and their oncology therapy included radiation in doses ranging from 50-80 Gy, delivered in 5 periods. In the test group, the patients mean age was 49.4 (standard deviation-sd-15.4) ranging 24-72. In the control group the mean age was 54.3 (sd 16.1) with a range between 24-75. Only one female was enrolled in the control group, and three in the test group. Three test patients and six control patients were smokers at the baseline.

\section{Methods}

Study design

The study was a parallel, double-blind, prospective, randomised clinical trial.

Patients were screened for compliance with the inclusion and exclusion criteria and once they agreed to participate by signing the IRB (Institutional Review Board) approved informed consent; they were entered in the study and appointed for the baseline visit.

The study consisted on three visits:

- Visit 1 or baseline: the day when radio-therapy was started.

- Visit 2: 14 days after baseline.

- Visit 3 or final visit: 28 days after baseline.

- Visit 1. Baseline. Patients were examined, outcome variables were recorded and samples for microbiology were collected. After this evaluation, all participating patients were randomised and the treatments were allocated by providing the assigned mouth rinse together with the instructions for use. They were then appointed for the next visit according to the study plan.

- Visit 2. 14-day evaluation. The same sampling and 
registration of outcome variables were done again, together with an interview with the patient assessing their compliance in using the assigned mouth rinse and the occurrence of any adverse event.

- Visit 3. Final visit after 28 days. Identical to visit 2, the sampling, registration of outcome variables, compliance and occurrence of any adverse event were carried out.

All the outcome variables were assessment by a single and calibrated examiner, who was blinded to the treatment assignment.

\section{Outcome variables}

\section{Evaluation of Mucositis}

The Scale of the Radiation Therapy Oncology Group/ European Organization Research and Treatment of Cancer (RTOG/EORTC) (16) was utilized. The clinical results have been described in other paper (15).

\section{Tongue samples}

Samples from the tongue dorsum were obtained by means of a tongue scrapper (Halita ${ }^{\circledR}$, Dentaid, Cerdanyola del Vallés, Spain), and a standardised loop of 0.5 $\mathrm{mm}$. The methods were identical to those described in (13). Briefly, the scrapper was used to take tongue coating from the dorsum of the tongue, and the loop was used to collect a standardised amount of the coating from the scrapper. This amount was transferred to a 1 mL Reduced Transport Fluid or RTF(17) vial and transported to the laboratory. At the laboratory, the sample was dispersed, serially diluted and inoculated on agar Saboreaud, to detect Candida species.

\section{Mucosa samples}

Samples from the buccal mucosa were obtained by gentle striking of the buccal mucosa in both sides with a cotton swab, for a few seconds. After that, the cotton swab was kept in its transport tube with $2 \mathrm{~mL}$ of RTF, and was transported to the laboratory, where the samples were processed as described for tongue samples.

Subgingival samples

Subgingival samples were collected from four selected sites. Sites with the worse clinical condition were selected, based on probing pocket depth and bleeding, considering also ease of access to avoid contamination, and usually mesio-buccal sites were selected. At sites, supragingival plaque was carefully removed to avoid bleeding using sterile gauze and / or curettes. Then, these sites were dried with sterile cotton rolls and gentle air drying. Two consecutive sterile paper points (medium size, Maillefer, Ballaigues, Switzerland) were inserted as deep as possible in the pocket, and left in place for 10 seconds. The paper points were transferred to a vial containing $1.5 \mathrm{~mL}$ of RTF, and pooled with all the other paper points. The vial was sent to the laboratory and processed within 24 hours. At the laboratory, vials were vortexed (30 seconds), serially diluted, and plated in two different media: Blood agar medium (No. 2 of Oxoid; Oxoid Ltd., Basingstoke, England), with
$5 \%$ horse blood, and haemin (5 mg/l) and menadione (1 $\mathrm{mg} / \mathrm{l}$ ) and Dentaid-1 medium (18). The blood agar plates were studied after 7 and 14 days of anaerobic incubation $\left(80 \% \mathrm{~N} 2 ; 10 \% \mathrm{H} 2 ; 10 \% \mathrm{CO}_{2}\right.$ at $\left.37^{\circ} \mathrm{C}\right)$; and the Dentaid-1 plates after $3-5$ days of $37^{\circ} \mathrm{C}$ incubation in air with $5 \%$ $\mathrm{CO}_{2}$. Plates were carefully examined for the identification of Aggregatibacter actinomycetemcomitans, Porphyromonas gingivalis, Prevotella intermedia/nigrescens, Tannerella forsythia, Parvimonas micra, Capnocytophaga sp., Eikenella corrodens and Fusobacterium sp., based on the morphology of the colony and using different standard biochemical tests to confirm the initial identification (RAPID ANA II). Other relevant colonies (those representing an important proportion of the flora) will be also isolated for further characterization. Colonies of each bacterial species will be counted, as will be the total number of colonies in a representative plate (between 30 to 300 colonies). Counts of A. actinomycetemcomitans will be performed on Dentaid-1 plates, based on its typical colony morphology, a catalase reaction and a set of specific enzymes. Additionally, any colony growing on Dentaid-1 medium, suspected of being an enteric rod, will be isolated. Dentaid-1 medium, as TSBV medium (19), have demonstrated excellent recovery of Enterobacteriaceae and Pseudomonadaceae species. Suspect non-oral, gram-negative, facultative anaerobic rods (20) will be subcultured on McConkey agar, purified and classified using a commercial identification kit system (API 20 E, Baxter Healthcare, West Sacramento, CA, USA). The panels and bacterial inoculae will be prepared following the recommendations of the manufacturer, and incubated for $18-24$ hours at $35^{\circ} \mathrm{C}$ in non-CO2 incubator. Bacterial speciation, based on 34 taxonomic test reactions, was performed using the software provided by the manufacturer.

\section{Treatments}

Patients included in the study were randomly assigned to one of the two treatments, either test or control. Randomisation was done through a computer-generated list that assigned treatments by numbers. Patients received a number after inclusion, corresponding to a numerically coded mouth rinse. The list and the numbered bottles were provided by the promoter, and the assignation of numbers was made by the researchers in consecutive order. Codes were not opened until the end of the study. Both patients and researchers were blinded throughout the study.

Patients in the test group rinsed with Perio-Aid Tratamiento ${ }^{\circledR}$ (Dentaid, Cerdanyola del Valles, Spain) composed of $0.12 \% \mathrm{CHX}$ and $0.05 \% \mathrm{CPC}$ as active ingredients. Patients in the control group rinsed with a placebo mouth rinse, identical to the test product but without the active components. Both formulations lacked any alcohol.

All patients received written instructions on the use of their assigned treatment. In brief, they should carry out 
their usual tooth-brushing and oral hygiene procedures, and then they should rinse with $15 \mathrm{~mL}$ of the assigned product, for 30 second, twice a day (morning and evening). Compliance was assessed by asking the patients to return the used bottles and by a brief interview.

Statistical analyses

The primary outcome variables were the presence/absence of pathogens (the most reliable microbiological variable in anaerobic culture) and the counts of Candida sp. in agar Saboreaud.

Microbiological variables were assessed either as intergroup changes, compared by means of the Mann-Whitney test, or intra-group changes, studied with the Wilcoxon test. The frequency of detection was compared by means of chi-square test.

Changes in the degree of mucositis were evaluated by means of the chi-square test with the Yates's correction. Comparisons between groups were assessed by means of the Mann-Whitney test.

The level of significance was established in $p<0.05$, and tendencies towards significance were considered for $\mathrm{p}<0.1$. No corrections for multiple comparisons were made.

The sample size was estimated, based on previous studies using microbiological outcome variables $(21,22)$, to be of, at least, 15 patients per arm.

\section{Results}

\section{Demographic data}

Demographic data of the population has been described in the Material and Methods section. No statistically significant differences were detected in demographic data between groups.

Detection of Candida spp. in mucosa (Table 1)
In mucosa samples, minor changes were detected in the control group throughout the whole study period. Conversely, reductions were observed in the test group, from baseline to 4 weeks $(p=0.05)$ and from 2 weeks to 4 weeks $(p=0.09)$ that showed a tendency towards statistical significance.

Detection of Candida spp. in tongue samples (Table 2) In tongue samples, a similar trend was found: minor changes or increases in the control group, while important reductions in the test group. However, none of the differences were statistically significant.

Microbiological findings in subgingival samples

Total bacterial counts decreased in both groups from baseline to the 2 -week visits, while minor changes occurred between 2 and 4 weeks. No statistically significant differences were detected both in intra- or intergroup comparisons (Table 3 ).

The most frequently isolated bacterial species at baseline were $F$. nucleatum $(68.8-85.7 \%), P$. intermedia (50.0-71.4\%) and $P$. gingivalis (42.9-43.8\%). They also represented the highest proportions of flora (Table 4).

$P$. gingivalis demonstrated a clear decrease in prevalence from baseline to 4 weeks $(\mathrm{p}=0.007)$ in the test group, while the placebo group did not show variations. Intergroup differences after 4 weeks were statistically significant $(\mathrm{p}=0.023)$.

$P$. intermedia showed an increase in frequency of detection in the placebo group, and minor changes in the test group, leading to significant differences in the intergroup comparison at the 4 -week visit $(\mathrm{p}=0.023)$.

A similar trend was observed for $C$. rectus, with significant reductions baseline- 4 weeks in the test group $(p=0.031)$. The reduction in the frequency of detection

Table 1. Candida sp. in mucosa samples (in colony forming units).

\begin{tabular}{|c|c|c|c|c|c|c|}
\hline CONTROL & baseline & week2 & week4 & base-w2 & base-w4 & w2-w4 \\
\hline $\mathrm{n}$ & 14 & 10 & 9 & 10 & 9 & 9 \\
\hline mean & 231 & 30 & 26 & 2 & -5 & -6 \\
\hline SD & 542 & 54 & 30 & 25 & 71 & 56 \\
\hline TEST & baseline & week2 & week4 & base-w2 & base-w4 & w2-w4 \\
\hline $\mathrm{n}$ & 16 & 12 & 11 & 12 & 11 & 11 \\
\hline mean & 715 & 684 & 38 & -7 & -716 & -708 \\
\hline SD & 1188 & 1178 & 62 & 1727 & 1195 & 1185 \\
\hline p value* & 0.327 & 0.765 & 0.760 & 0.448 & 0.175 & 0.131 \\
\hline CONTROL & \multicolumn{3}{|c|}{ p value $* *$} & $>0.999$ & 0.734 & 0.734 \\
\hline TEST & \multicolumn{3}{|c|}{$\mathrm{p}$ value $* *$} & 0.844 & 0.055 & 0.098 \\
\hline
\end{tabular}

* Mann-Whitney test for intergroup comparison.

** Wilcoxon matched-pairs signed-ranks for intragroup comparison.

Base-baseline, 2w-2 weeks, 4w-4 weeks, SD-standard deviation.

Base-w2, base-w4 and w2-w4, represents changes between visits, with positive values meaning increase and negative values decrease. 
Table 2. Candida sp. in tongue samples (in colony forming units).

\begin{tabular}{|c|c|c|c|c|c|c|}
\hline CONTROL & baseline & week2 & week4 & base-w 2 & base-w4 & w2-w4 \\
\hline $\mathrm{n}$ & 12 & 10 & 9 & 9 & 8 & 9 \\
\hline mean & 17 & 45 & 136 & 33 & 135 & 86 \\
\hline $\mathrm{SD}$ & 29 & 115 & 377 & 118 & 393 & 258 \\
\hline TEST & baseline & week2 & week4 & base-w2 & base-w4 & w2-w4 \\
\hline $\mathrm{n}$ & 15 & 12 & 9 & 11 & 8 & 9 \\
\hline mean & 682 & 376 & 85 & -527 & -1102 & -324 \\
\hline $\mathrm{SD}$ & 1826 & 882 & 159 & 2379 & 2494 & 1032 \\
\hline $\mathrm{p}$ value* & $>0.999$ & 0.974 & 0.505 & 0.568 & 0.279 & 0.354 \\
\hline CONTROL & \multicolumn{3}{|c|}{$\mathrm{p}$ value $* *$} & 0.195 & 0.547 & 0.945 \\
\hline TEST & \multicolumn{3}{|c|}{$\mathrm{p}$ value $* *$} & 0.945 & 0.312 & 0.312 \\
\hline
\end{tabular}

* Mann-Whitney test for intergroup comparison.

** Wilcoxon matched-pairs signed-ranks for intragroup comparison.

Base-baseline, 2w-2 weeks, 4w-4 weeks, sd-standard deviation.

Base-w2, base-w4 and w2-w4, represents changes between visits, with positive values meaning increase and negative values decrease.

Table 3. Subgingival samples: total log of colony forming units expressed as mean and standard deviation (SD) per visit and in changes between visits.

\begin{tabular}{|c|c|c|c|c|c|c|c|}
\hline & & baseline & week 2 & week 4 & $\begin{array}{c}\text { baseline- } \\
\text { week } 2 \\
\end{array}$ & $\begin{array}{c}\text { baseline- } \\
\text { week } 4\end{array}$ & $\begin{array}{l}\text { week 2- } \\
\text { week } 4\end{array}$ \\
\hline \multirow{3}{*}{ placebo } & $\mathrm{n}$ & 12 & 10 & 9 & 8 & 7 & 9 \\
\hline & mean & 6.829 & 6.192 & 6.278 & -0.659 & -0.427 & 0.193 \\
\hline & SD & 0.544 & 0.874 & 0.71 & 0.762 & 0.526 & 0.616 \\
\hline & & & & & & & \\
\hline \multirow{3}{*}{ test } & $\mathrm{n}$ & 13 & 11 & 9 & 8 & 7 & 9 \\
\hline & mean & 6.331 & 5.745 & 5.906 & -0.093 & -0.017 & -0.006 \\
\hline & $\mathrm{SD}$ & 1.079 & 0.928 & 0.89 & 0.904 & 0.596 & 0.537 \\
\hline inter group & t-test & 0.165 & 0.271 & 0.342 & 0.197 & 0.198 & 0.477 \\
\hline
\end{tabular}

of $E$. corrodens was also statistically significant in the test group both after 2 weeks $(\mathrm{p}=0.021)$ and after 4 weeks $(p=0.034)$.

Minor changes with non significant differences were detected for other bacterial species.

No clear trends were observed for mean counts and mean proportions of flora of bacterial species (Table 4).

No overgrowth of other opportunistic species was detected.

Adverse effects

No relevant adverse effects were reported in any group.

\section{Discussion}

The results from the present study have shown some relevant microbiological effects when using a non-alcohol, CHX and CPC based mouth rinse, in patients undergo- ing radiation therapy as part of the treatment of a headand-neck cancer. These results, however, should be interpreted with caution, due to the limited sample size and the limitations of its calculation, the heterogeneity of the patient sample (including smoking habit) and the inherent difficulties when enrolling patients with severe health conditions in clinical trials. In addition, no clinical effects (15) were associated to the improvements in the microbiological variables.

There are many confounding factors that may have influenced the results, such as: the total radiation dose and treatment regimen; the heterogeneity of the patient sample due to differences in tumour type, clinical stage, histology, location, extension, etc, and the existence of previous or concomitant chemotherapy. Moreover, the deterioration of the patient systemic status during the 
Table 4. Subgingival samples: colony forming units (cfu), proportions of flora and frequency of detection of different periodontal pathogens at every study visit.

\begin{tabular}{|c|c|c|c|c|c|c|c|c|c|c|}
\hline \multirow[b]{2}{*}{ group } & \multirow[b]{2}{*}{ variable } & \multicolumn{3}{|c|}{ A. actinomycetemcomitans } & \multicolumn{3}{|c|}{ P.gingivalis } & \multicolumn{3}{|c|}{ P.intermedia } \\
\hline & & baseline & week 2 & week 4 & baseline & week 2 & week 4 & baseline & week 2 & week 4 \\
\hline \multirow{5}{*}{ PLACEBO } & n samples & 14 & 10 & 9 & 14 & 10 & 9 & 14 & 10 & 9 \\
\hline & mean cfu & 4714 & 0 & 0 & 161700 & 210540 & 742867 & 398451 & 126720 & 316360 \\
\hline & mean proportion $(\%)$ & 0.04 & 0.00 & 0.00 & 6.85 & 5.70 & 10.46 & 4.05 & 4.84 & 4.39 \\
\hline & positive samples & 1 & 0 & 0 & 6 & 4 & 4 & 10 & 6 & 9 \\
\hline & frequency of detection & $7.1 \%$ & $0.0 \%$ & $0.0 \%$ & $42.9 \%$ & $40.0 \%$ & $44.4 \%$ & $71.4 \%$ & $60.0 \%$ & $100.0 \%$ \\
\hline \multirow{5}{*}{ TEST } & n samples & 16 & 12 & 11 & 16 & 12 & 11 & 16 & 12 & 11 \\
\hline & mean cfu & 0 & 7260 & 684 & 178679 & 144870 & 0 & 185076 & 137830 & 223320 \\
\hline & mean proportion (\%) & 0.00 & 0.05 & 0.02 & 0.65 & 1.41 & 0.00 & 0.71 & 3.64 & 3.61 \\
\hline & positive samples & 0 & 1 & 1 & 7 & 4 & 0 & 8 & 9 & 5 \\
\hline & frequency of detection & $0.0 \%$ & $8.3 \%$ & $9.1 \%$ & $43.8 \%$ & $33.3 \%$ & $0.0 \%$ & $50.0 \%$ & $75.0 \%$ & $45.5 \%$ \\
\hline & & \multicolumn{3}{|c|}{ P.micra } & \multicolumn{3}{|c|}{ F.nucleatum } & \multicolumn{3}{|c|}{ C.rectus } \\
\hline group & variable & baseline & week 2 & week 4 & baseline & week 2 & week 4 & baseline & week 2 & week 4 \\
\hline \multirow{5}{*}{ PLACEBO } & n samples & 14 & 10 & 9 & 14 & 10 & 9 & 14 & 10 & 9 \\
\hline & mean cfu & 4714 & 21120 & 35200 & 480716 & 184708 & 156127 & 0 & 660 & 4400 \\
\hline & mean proportion $(\%)$ & 0.31 & 0.98 & 0.93 & 6.00 & 6.51 & 4.91 & 0.00 & 0.03 & 0.31 \\
\hline & positive samples & 2 & 3 & 3 & 12 & 10 & 9 & 0 & 1 & 3 \\
\hline & frequency of detection & $14.3 \%$ & $30.0 \%$ & $33.3 \%$ & $85.7 \%$ & $100.0 \%$ & $100.0 \%$ & $0.0 \%$ & $10.0 \%$ & $33.3 \%$ \\
\hline \multirow{5}{*}{ TEST } & $\mathrm{n}$ samples & 16 & 12 & 11 & 16 & 12 & 11 & 16 & 12 & 11 \\
\hline & mean cfu & 13613 & 2145 & 13140 & 273137 & 48351 & 86220 & 1246 & 550 & 1200 \\
\hline & mean proportion $(\%)$ & 0.23 & 1.77 & 2.57 & 2.09 & 4.75 & 3.14 & 0.07 & 0.03 & 0.03 \\
\hline & positive samples & 3 & 3 & 4 & 11 & 10 & 7 & 3 & 1 & 1 \\
\hline & frequency of detection & $18.8 \%$ & $25.0 \%$ & $36.4 \%$ & $68.8 \%$ & $83.3 \%$ & $63.6 \%$ & $18.8 \%$ & $8.3 \%$ & $9.1 \%$ \\
\hline & & \multicolumn{3}{|c|}{ E.corrodens } & \multicolumn{3}{|c|}{ T.forsythia } & \multicolumn{3}{|c|}{ Capnocytophaga sp. } \\
\hline group & variable & baseline & week 2 & week 4 & baseline & week 2 & week 4 & baseline & week 2 & week 4 \\
\hline \multirow{5}{*}{ PLACEBO } & n samples & 14 & 10 & 9 & 14 & 10 & 9 & 14 & 10 & 9 \\
\hline & mean cfu & 23760 & 726 & 29333 & 10513 & 0 & 10267 & 1933 & 2640 & 0 \\
\hline & mean proportion $(\%)$ & 0.59 & 0.10 & 0.25 & 0.30 & 0.00 & 1.02 & 0.07 & 0.12 & 0.00 \\
\hline & positive samples & 2 & 2 & 1 & 2 & 0 & 2 & 3 & 2 & 0 \\
\hline & frequency of detection & $14.3 \%$ & $20.0 \%$ & $11.1 \%$ & $14.3 \%$ & $0.0 \%$ & $22.2 \%$ & $21.4 \%$ & $20.0 \%$ & $0.0 \%$ \\
\hline \multirow{5}{*}{ TEST } & $\mathrm{n}$ samples & 16 & 12 & 11 & 16 & 12 & 11 & 16 & 12 & 11 \\
\hline & mean cfu & 2500 & 0 & 0 & 0 & 0 & 0 & 28875 & 0 & 0 \\
\hline & mean proportion $(\%)$ & 0.22 & 0.00 & 0.00 & 0.00 & 0.00 & 0.00 & 0.52 & 0.00 & 0.00 \\
\hline & positive samples & 5 & 0 & 0 & 0 & 0 & 0 & 2 & 0 & 0 \\
\hline & frequency of detection & $31.3 \%$ & $0.0 \%$ & $0.0 \%$ & $0.0 \%$ & $0.0 \%$ & $0.0 \%$ & $12.5 \%$ & $0.0 \%$ & $0.0 \%$ \\
\hline
\end{tabular}

oncology therapy, precluded some patients from continuing the study and therefore, to comply with the programmed study visits.

Previous studies have reported changes in the oral microflora as a consequence of irradiation therapy, including an increase in the detection of Candida $s p .(2,4,23)$, an increase in oral colonization of aerobic and anaerobic gram-negative rods and cocci (5), an increase in the detection of caries-associated micro-organisms $(7,8,9)$, and changes in the subgingival microflora with an increase in the presence of non-oral micro-organisms $(3,6)$. In the present study, the use of the evaluated mouth rinse with CHX and CPC, was associated with significant microbiological benefits, both at subgingival (effects on P. gin- givalis, $P$. intermedia, C. rectus, E. corrodens), tongue (Candida sp.), and mucosal (Candida sp.) samples. A similar impact, although not statistically significant was reported by Ferretti et al. (21) in the group of 30 irradiated patients, with a reduction in oral streptocci and yeasts in the group using $0.12 \% \mathrm{CHX}$, t.i.d. Conversely, in other study also with 30 patients, the group using $0.1 \% \mathrm{CHX}$, q.i.d., had similar colonization patterns that the control group in terms of Candida sp., staphylococci, and other super infecting microorganisms (22). One of the explanations for the positive results in our study could be related to the improved formulation of the tested product, thus providing higher activity $(11,12)$.

In the present study, three oral niches were samples, the 
subgingival niche for the evaluation of anaerobic bacteria and the tongue and saliva for the evaluation of $\mathrm{Candi-}$ $d a$ sp. The evaluation of the subgingival microflora was selected in order to provide a representative overview of the presence of anaerobic bacteria in the mouth, since most anaerobic bacteria have their primary site in this area and their subgingival presence is associated with its presence in other intraoral sites $(13,24)$. Anaerobic bacteria could be related with worsen of the mucositis lesions by contamination, especially in the ulcerative phase (25). Conversely, the subgingival area is not the primary site for Candida sp. and other intraoral sites were evaluated to assess its presence.

The impact of the use of the evaluated mouth rinse in the detection of Candida sp. may suggest that CHX mouth rinse could be a reasonable alternative in the prevention of candidiasis in risk patients. Some authors have also suggested its use in the treatment for oral fungal infections, as alternative for nystatin rinses, clotrimazole or ketoconazole (26).

It is clear that the potential benefit of this preventive regime using an antimicrobial rinse relies more in the control of oral micro-organisms and in the reduction in oropharyngeal candidosis, rather than on a direct effect upon oral mucositis.

\section{Conclusions}

Within the limitations of the small sample size, this study suggests that the use of a $0.12 \% \mathrm{CHX}$ and $0.05 \%$ CPC mouth rinse may lead to improvements in microbiological parameters in patients irradiated for headand-neck cancer.

\section{References}

References with links to Crossref - DOI

1. Scully C, Sonis S, Diz PD. Oral mucositis. Oral Dis. 2006;12:229-41. 2. Jham BC, França EC, Oliveira RR, Santos VR, Kowalski LP, da Silva Freire AR. Candida oral colonization and infection in Brazilian patients undergoing head and neck radiotherapy: a pilot study. Oral Surg Oral Med Oral Pathol Oral Radiol Endod. 2007;103:355-8.

3. Leung WK, Jin LJ, Samaranayake LP, Chiu GK. Subgingival microbiota of shallow periodontal pockets in individuals after head and neck irradiation. Oral Microbiol Immunol. 1998;13:1-10.

4. Thaweboon S, Thaweboon B, Srithavaj T, Choonharuangdej S. Oral colonization of Candida species in patients receiving radiotherapy in the head and neck area. Quintessence Int. 2008;39:e52-7.

5. Leung WK, Jin LJ, Yam WC, Samaranayake LP. Oral colonization of aerobic and facultatively anaerobic gram-negative rods and cocci in irradiated, dentate, xerostomic individuals. Oral Microbiol Immunol. 2001;16:1-9.

6. Almståhl A, Wikström M, Fagerberg-Mohlin B. Microflora in oral ecosystems in subjects with radiation-induced hyposalivation. Oral Dis. 2008;14:541-9.

7. Al-Nawas B, Grötz KA. Prospective study of the long term change of the oral flora after radiation therapy. Support Care Cancer. 2006;14:291-6.

8. Tong HC, Gao XJ, Dong XZ. Non-mutans streptococci in patients receiving radiotherapy in the head and neck area. Caries Res. 2003;37:261-6.

9. Srithavaj T, Thaweboon S. Determination of oral microflora in irradiated ocular deformed children. Southeast Asian J Trop Med Public Health. 2006;37:991-5.
10. Ellepola AN, Samaranayake LP. Adjunctive use of chlorhexidine in oral candidoses: a review. Oral Dis. 2001;7:11-7.

11. Herrera D, Roldán S, Santacruz I, Santos S, Masdevall M, Sanz M. Differences in antimicrobial activity of four commercial $0.12 \%$ chlorhexidine mouthrinse formulations: an in vitro contact test and salivary bacterial counts study. J Clin Periodontol. 2003;30:307-14. 12. Quirynen M, Avontroodt P, Peeters W, Pauwels M, Coucke W, van Steenberghe D. Effect of different chlorhexidine formulations in mouthrinses on de novo plaque formation. J Clin Periodontol. 2001;28:1127-36

13. Roldán S, Winkel EG, Herrera D, Sanz M, Van Winkelhoff AJ. The effects of a new mouthrinse containing chlorhexidine, cetylpyridinium chloride and zinc lactate on the microflora of oral halitosis patients: a dual-centre, double-blind placebo-controlled study. J Clin Periodontol. 2003;30:427-34.

14. Santos S, Herrera D, López E, O’Connor A, González I, Sanz M. A randomized clinical trial on the short-term clinical and microbiological effects of the adjunctive use of a $0.05 \%$ chlorhexidine mouth rinse for patients in supportive periodontal care. J Clin Periodontol. 2004;31:45-51.

15. Lanzós I, Herrera D, Santos S, O’Connor A, Peña C, Lanzós E, et al. Mucositis in irradiated cancer patients: effects of an antiseptic mouthrinse. Med Oral Patol Oral Cir Bucal. 2010;15:e732-8.

16. Cox JD, Stetz J, Pajak TF. Toxicity criteria of the Radiation Therapy Oncology Group (RTOG) and the European Organization for Research and Treatment of Cancer (EORTC). Int J Radiat Oncol Biol Phys. 1995;31:1341-6.

17. Syed SA, Loesche WJ. Survival of human dental plaque flora in various transport media. Appl Microbiol. 1972;24:638-44.

18. Alsina M, Olle E, Frias J. Improved, low-cost selective culture medium for Actinobacillus actinomycetemcomitans. J Clin Microbiol. 2001;39:509-13.

19. Slots J. Selective medium for isolation of Actinobacillus actinomycetemcomitans. J Clin Microbiol. 1982;15:606-9.

20. Slots J, Feik D, Rams TE. Prevalence and antimicrobial susceptibility of Enterobacteriaceae, Pseudomonadaceae and Acinetobacter in human periodontitis. Oral Microbiol Immunol. 1990;5:149-54.

21. Ferretti GA, Raybould TP, Brown AT, Macdonald JS, Greenwood M, Maruyama Y, et al. Chlorhexidine prophylaxis for chemotherapyand radiotherapy-induced stomatitis: a randomized double-blind trial. Oral Surg Oral Med Oral Pathol. 1990;69:331-8.

22. Spijkervet FK, van Saene HK, Panders AK, Vermey A, van Saene JJ, Mehta DM, et al. Effect of chlorhexidine rinsing on the oropharyngeal ecology in patients with head and neck cancer who have irradiation mucositis. Oral Surg Oral Med Oral Pathol. 1989;67:154-61. 23. Kuten A, Ben-Aryeh H, Berdicevsky I, Ore L, Szargel R, Gutman $\mathrm{D}$, et al. Oral side effects of head and neck irradiation: correlation between clinical manifestations and laboratory data. Int J Radiat Oncol Biol Phys. 1986;12:401-5.

24. Socransky SS, Haffajee AD. Periodontal microbial ecology. Periodontol 2000. 2005;38:135-87.

25. Sonis ST, Elting LS, Keefe D, Peterson DE, Schubert M, HauerJensen M, et al. Perspectives on cancer therapy-induced mucosal injury: pathogenesis, measurement, epidemiology, and consequences for patients. Cancer. 2004;100:1995-2025.

26. Hancock PJ, Epstein JB, Sadler GR. Oral and dental management related to radiation therapy for head and neck cancer. J Can Dent Assoc. 2003;69:585-90.

\section{Acknowledgements}

The authors want to thank the personnel working at the Oncological Radiotherapy Service "Hospital 12 de Octubre" (Madrid, Spain).

Source of Funding

The study was supported by a research grant (contract between University Complutense and Dentaid).

\section{Conflict of Interests}

The authors declare that they have no conflict of interests.

Supported by a research grant from Dentaid (Cerdanyola del Vallés, Spain) 